\title{
Galaxy Zoo: Outreach and Science Hand in Hand
}

\author{
Karen L. Masters ${ }^{1,2}$ and the Galaxy Zoo Team \\ ${ }^{1}$ Institute for Cosmology and Gravitation, University of Portsmouth, Dennis Sciama Building, \\ Burnaby Road, Portsmouth, PO1 3FX, UK \\ email: karen.masters@port.ac.uk \\ ${ }^{2}$ South East Physics Network, www.sepnet.ac.uk
}

\begin{abstract}
Galaxy Zoo (www.galaxyzoo.org) is familiar to many as a hugely successful public engagement project. Hundreds of thousands of members of the public have contributed to Galaxy Zoo which collects visual classifications of galaxies in Sloan Digital Sky Survey and Hubble Space Telescope images. Galaxy Zoo has inspired a suite of similar Citizen Science projects known as "The Zooniverse" (www.zooniverse.org) which now has well over half a million participants. Galaxy Zoo has also shown itself, in a series of peer reviewed papers, to be a fantastic database for the study of galaxy evolution. In this invited talk I described how that public engagement via citizen science is not only an effective means of outreach from data intensive surveys, but if done right can and must also increase the scientific output of the survey.
\end{abstract}

\section{Introduction}

Galaxy Zoo was launched in July 2007 to solve the problem of how to visually classify the $\sim 1$ million galaxies in the Sloan Digital Sky Survey Main Galaxy Sample (Strauss et al. 2002). The original site asked volunteers to classify galaxies as either spiral or elliptical - the most basic morphological split. Something in Galaxy Zoo resonated extraordinarily with the general public. By April 2008, when the Galaxy Zoo team submitted their first paper (Lintott et al.2008), over 100,000 volunteers had classified each of the images an average of 38 times. $\dagger$ The popularity of Galaxy Zoo and the number of classifications received enabled science which simply would not have been possible otherwise. Not only does Galaxy Zoo give a classification for each galaxy, but also by collecting $\sim 40$ independent classifications of each galaxy out can produce an estimate of how reliable that classification is, and calibrate individual clicks with weightings to remove volunteers who disagree with most other classifiers. Galaxy Zoo was so popular that it is now in its fourth version, asking for detailed classifications of both SDSS and HST images of galaxies.

The scientific results coming out from Galaxy Zoo have been essential to its success. A survey which studied the motivations of the citizen scientists participating in Galaxy Zoo, (Raddick et al. 2010) showed that the majority of people identified a desire to help with scientific research as their main motivation for spending time on the site. Galaxy Zoo was designed with specific and immediate questions to answer. The first publications from Galaxy Zoo came out within a year of launch (Lintott et al. 2008, Land et al. 2008), and the total number of peer reviewed papers based on Galaxy Zoo data is now more than 30 (e.g see Table 1 of Masters 2012). They include among them work separating colour and morphology in the galaxy population (e.g. Bamford et al. 2009, Skibba et al. 2009,

\footnotetext{
$\dagger$ All of the data from this first phase of Galaxy Zoo were published in Lintott et al. (2011) and are available to download via the SDSS servers at http://skyserver.sdss3.org/CasJobs/. For more information on the history of the Galaxy Zoo project see Masters (2012) in this volume, or Fortson et al. (2012).
} 
Schawinski et a. 2009, Masters et al. 2010a,b), on the interplay between supermassive black hole activity and host galaxies (e.g. Lintott et al. 2009, Schawinski et al. 2010a,b, Teng et al. 2012, Simmons et al. 2012) and on the role bars play on galaxy evolution (e.g. Masters et al. 2011, Hoyle et al. 2011, Skibba et al. 2012, Masters et al. 2012). Galaxy Zoo classifications have also been used to calibrate machine learning and other automated methods of galaxy classification, illustrating a complementarity with these methods which will become even more important as galaxy catalogues grow even larger (e.g. Banerji et al. 2010).

Galaxy Zoo was a pioneer in what has become a new methodology of involving "citizen scientists" in research. The Zooniverse $\nmid$ was launched in December 2009 to provide a framework to collect a variety of similar projects. This now includes several other astronomically themed projects. A review of citizen science (including Zooniverse project) contributions to astronomy research can be found at Christian et al. (2012).

Astronomers (and other scientists) involved in big data projects can now propose to develop their own Zooniverse projectł. The philosophy of citizen science in the Zooniverse has been built based on the experience with Galaxy Zoo. Central to this is that all projects in the Zooniverse exist to analyse data and produce science (or research) which would not be possible without the help of citizen scientists.

Involving the public in the scientific method in this way provides a fantastic opportunity for science education across a wide variety of audiences and at many levels, but scientists embarking on a Zooniverse project must commit resources to produce scientific results out of the contributions. We can all benefit when we work together.

\section{Acknowledgements}

This publication has been made possible by the participation of more than 200,000 volunteers in the Galaxy Zoo project. Their contributions are individually acknowledged at http://www.galaxyzoo.org/volunteers.

KLM acknowledges funding from the Peter and Patricia Gruber Foundation as the 2008 Peter and Patricia Gruber Foundation IAU Fellow, and from a 2010 Leverhulme Trust Early Career Fellowship, as well as support from the Royal Astronomical Society to attend the 28th GA of the IAU.

The Citizen Science Alliance acknowledges funding from the Alfred P. Sloan Foundation to enable open calls for Zooniverse projects.

\section{References}

Bamford, S. P., et al. 2009, MNRAS, 393, 1324

Banerji, M., Lahav, O., Lintott, C. J., et al. 2010, MNRAS, 406, 342

Christian, C., Lintott, C., Smith, A., Fortson, L., \& Bamford, S. 2012, Organizations, People and Strategies in Astronomy Vol. 1, Edited by Andre Heck, Venngeist, Duttlenheim (2012) pp. 183-197, 183 (arXiv:1202.2577)

Fortson, L., Masters, K., Nichol, R., et al. 2012, Advances in Machine Learning and Data Mining for Astronomy, CRC Press, Eds.: Michael J. Way et al., p. 213-236, 213 (arXiv:1104.5513)

Hoyle, B., Masters, K. L., Nichol, R. C., et al. 2011, MNRAS, 415, 3627

Land, K., Slosar, A., Lintott, C. J., et al. 2008, MNRAS, 388, 1686

Lintott, C. J., et al. 2008, MNRAS, 389, 1179

Lintott, C. J., Schawinski, K., Keel, W., et al. 2009, MNRAS 399, 129

Lintott, C. J., et al. 2011, MNRAS, 410, 166

Masters, K. L., et al. 2010a, MNRAS 404, 792.

$\dagger$ www.zooniverse.org

$\ddagger$ See http://www.citizensciencealliance.org/proposals.html 
Masters, K. L., et al. 2010b, MNRAS 405, 783.

Masters, K. L., et al. 2011, MNRAS, 411, 2026

Masters, K. L., Nichol, R. C., Haynes, M. P., et al. 2012, MNRAS, 424, 2180

Masters, K. L. 2012, Highlights of Astronomy, Volume 16, Ed Thierry Montmerle.

Raddick, M. J., Bracey, G., Gay, P. L., et al. 2010, Astronomy Education Review, 9, 010103

Skibba, R. A., et al. 2009, MNRAS, 399, 966

Skibba, R. A., Masters, K. L., Nichol, R. C., et al. 2012, MNRAS, 423, 1485

Schawinski, K., Evans, D. A., Virani, S., et al. 2010a, ApJL, 724, L30

Schawinski, K., Urry, C. M., Virani, S., et al. 2010b, ApJ, 711, 284

Simmons, B. D., Lintott, C., Schawinski, K., et al. 2012, MNRAS (submitted; arXiv:1207.4190)

Strauss, M. A., et al. 2002, AJ, 124, 1810

Teng, S. H., Schawinski, K., Urry, C. M., et al. 2012, ApJ, 753, 165 